PROCEEDINGS OF THE

AMERICAN MATHEMATICAL SOCIETY

Volume 137, Number 12, December 2009, Pages 4025-4028

S 0002-9939(09)10021-7

Article electronically published on July 22, 2009

\title{
ON THE DIVISIBILITY OF THE CLASS NUMBER OF IMAGINARY QUADRATIC NUMBER FIELDS
}

\author{
STÉPHANE R. LOUBOUTIN
}

(Communicated by Ken Ono)

\begin{abstract}
We prove that if at least one of the prime divisors of an odd integer $U \geq 3$ is equal to $3 \bmod 4$, then the ideal class group of the imaginary quadratic field $\mathbf{Q}\left(\sqrt{1-4 U^{n}}\right)$ contains an element of order $n$.
\end{abstract}

\section{INTRODUCTION}

In 1953, N. C. Ankeny and S. Chowla proved in AC that for any given integer $n>1$ there are infinitely many imaginary quadratic number fields of the form $\mathbf{Q}\left(\sqrt{x^{2}-3^{n}}\right)$, with $x^{2}-3^{n}$ square-free, whose class numbers are divisible by $n$. In 2008, Y. Kishi came back in Ki to this family and proved that in the case where $x=4^{k}<3^{n}$ is a power of 4 , then $n$ always divides the class number of $\mathbf{Q}\left(\sqrt{x^{2}-3^{n}}\right)$. In 1978, B. H. Gross and D. E. Rohrlich delineated in GR a proof of the fact that for any given odd $n>1$ there are infinitely many imaginary quadratic number fields whose class numbers are divisible by $n$, namely the imaginary quadratic fields $\mathbf{Q}\left(\sqrt{1-4 U^{n}}\right), U>1$. In 2002, J. H. E. Cohn came back in [Co] to this family and proved that $n \neq 4$, be it odd or even, always divides the class number of the imaginary quadratic fields $\mathbf{Q}\left(\sqrt{1-4 \cdot 2^{n}}\right)$. The aim of this note is (i) to expound Gross and Rohrlich's proof (the first point of Theorem 11) and (ii) to prove a result stronger than GR, Theorem 5.3] (the second point of Theorem (1). However, we note that the family of imaginary quadratic number fields $\mathbf{Q}\left(\sqrt{1-4 U^{n}}\right)$ is much thinner than the families studied in [Mur] or [So]. Thus, our results, though pleasingly explicit, have no hope of approaching a proof of the Cohen-Lenstra heuristics, which state that the number $N_{n}(x)$ of imaginary quadratic fields $\mathbf{Q}(\sqrt{-d})$ with $0<d<x, d$ square-free, whose class groups contain elements of order $n$, is asymptotic to $C_{n} x$ as $x$ tends to infinity, for some positive and explicit constant $C_{n}$.

Theorem 1. Fix $n>1$. Then:

1. If $n$ is odd, then for any integer $U \geq 2$ the ideal class groups of the imaginary quadratic fields $\mathbf{Q}\left(\sqrt{1-4 U^{n}}\right)$ contain an element of order $n$.

2. If at least one of the prime divisors of an odd integer $U \geq 3$ is equal to $3 \bmod 4$, then the ideal class group of the imaginary quadratic field $\mathbf{Q}\left(\sqrt{1-4 U^{n}}\right)$ contains an element of order $n$.

Received by the editors March 20, 2009, and, in revised form, April 9, 2009.

2000 Mathematics Subject Classification. Primary 11R29; Secondary 11R11.

Key words and phrases. Class number, imaginary quadratic field, divisibility.

(C)2009 American Mathematical Society Reverts to public domain 28 years from publication 


\section{BEING A $p$ TH POWER}

Throughout this note, we let $\mathbf{k}$ be a quadratic number field. Let $\sigma$ be its nontrivial $\mathbf{Q}$-automorphism. Let $\mathbf{A}_{\mathbf{k}}$ be its ring of algebraic integers and let $d_{\mathbf{k}}$ be its discriminant. Let $\operatorname{Tr}(\alpha)=\alpha+\sigma(\alpha) \in \mathbf{Z}$ and $N(\alpha)=\alpha \sigma(\alpha) \in \mathbf{Z}$ be the trace and norm of $\alpha \in \mathbf{A}_{\mathbf{k}}$. Let $\mathbf{A}_{\mathbf{k}}{ }^{*}$ be the group of units of $\mathbf{A}_{\mathbf{k}}$. We say that $\alpha \in \mathbf{A}_{\mathbf{k}}$ is associated with $\beta \in \mathbf{A}_{\mathbf{k}}$ if there exists $\eta \in \mathbf{A}_{\mathbf{k}}{ }^{*}$ such that $\beta=\eta \alpha$, i.e. if the principal ideal $(\beta)$ of $\mathbf{A}_{\mathbf{k}}$ is equal to $(\alpha)$.

Lemma 2. Let $\alpha \in \mathbf{A}_{\mathbf{k}}$. If $k \geq 1$ is odd, then $\operatorname{Tr}(\alpha)$ divides $\operatorname{Tr}\left(\alpha^{k}\right)$. If $p \geq 3$ is prime, then $\operatorname{Tr}\left(\alpha^{p}\right) \equiv \operatorname{Tr}(\alpha)(\bmod p)$. Hence, if $\operatorname{Tr}(\alpha)=1$ and if $\alpha$ is a pth power in $\mathbf{A}_{\mathbf{k}}$, then $\alpha$ is a unit of $\mathbf{A}_{\mathbf{k}}$.

Proof. Since $\alpha^{2}-\operatorname{Tr}(\alpha) \alpha+N(\alpha)=0$ with $\operatorname{Tr}(\alpha) \in \mathbf{Z}$ and $N(\alpha) \in \mathbf{Z}$, we have $\alpha^{k+2}=\operatorname{Tr}(\alpha) \alpha^{k+1}-N(\alpha) \alpha^{k}$ for $k \geq 0$, which, in taking the trace, yields the first result by induction on $k \geq 1$ odd. Since $p$ divides the binomial coefficients $\left(\begin{array}{l}p \\ l\end{array}\right)$ for $1 \leq l \leq p-1$, we have $\operatorname{Tr}(\alpha)^{p}-\operatorname{Tr}\left(\alpha^{p}\right)=(\alpha+\sigma(\alpha))^{p}-\alpha^{p}-\sigma(\alpha)^{p}=$ $\sum_{l=1}^{p-1}\left(\begin{array}{l}p \\ l\end{array}\right) \alpha^{l} \sigma(\alpha)^{p-l} \in p \mathbf{A}_{\mathbf{k}} \cap \mathbf{Z}=p \mathbf{Z}$ and, using $\operatorname{Tr}(\alpha)^{p} \equiv \operatorname{Tr}(\alpha)(\bmod p)$, we obtain the second result. If $\alpha=\left(1+y \sqrt{d_{\mathbf{k}}}\right) / 2=\beta^{p}=\left(\left(u+v \sqrt{d_{\mathbf{k}}}\right) / 2\right)^{p}$ of trace equal to 1 is a $p$ th power in $\mathbf{A}_{\mathbf{k}}$, then $u=\operatorname{Tr}(\beta)$ divides $1=\operatorname{Tr}(\alpha)=\operatorname{Tr}\left(\beta^{p}\right)$ (the first point), hence $u= \pm 1$, and $u=\operatorname{Tr}(\beta) \equiv \operatorname{Tr}\left(\beta^{p}\right)=\operatorname{Tr}(\alpha)=1(\bmod p)$ (the second point), hence $u=1$. Then $\beta=\left(1+v \sqrt{d_{\mathbf{k}}}\right) / 2 \in \mathbf{A}_{\mathbf{k}}$ satisfies $\sigma(\beta)=1-\beta$; hence $\beta^{p}+(1-\beta)^{p}=\operatorname{Tr}\left(\beta^{p}\right)=\operatorname{Tr}(\alpha)=1$ and

$$
0=(1-\beta)^{p}-1+\beta^{p}=\sum_{l=1}^{p-1}\left(\begin{array}{l}
p \\
l
\end{array}\right)(-\beta)^{l}=-p \beta\left(1-\beta \sum_{l=2}^{p-1} \frac{1}{p}\left(\begin{array}{l}
p \\
l
\end{array}\right)(-\beta)^{l-2}\right) .
$$

Therefore, we have

$$
\beta \sum_{l=2}^{p-1} \frac{1}{p}\left(\begin{array}{l}
p \\
l
\end{array}\right)(-\beta)^{l-2}=1
$$

and $\beta$, hence $\alpha$, is a unit of $\mathbf{A}_{\mathbf{k}}$.

Proposition 3. Let $\mathbf{k}$ be an imaginary quadratic field. If $\alpha \in \mathbf{A}_{\mathbf{k}}$ with $\operatorname{Tr}(\alpha)=1$ is associated with a pth power for some odd prime $p \geq 3$, then $\alpha$ is a unit of $\mathbf{A}_{\mathbf{k}}$.

Proof. If $\mathbf{k} \neq \mathbf{Q}(\sqrt{-3})$, or if $\mathbf{k}=\mathbf{Q}(\sqrt{-3})$ and $p>3$, then any unit in $\mathbf{k}$ is a $p$ th power, and the result follows from Lemma 2. Now assume that $\mathbf{k}=\mathbf{Q}(\sqrt{-3})$ and $p=3$. Set $j=(-1+\sqrt{-3}) / 2$. We have $\alpha=\beta^{3}, j \beta^{3}$ or $j^{2} \beta^{3}$ in $\mathbf{A}_{\mathbf{k}}$, and $\alpha \neq \beta^{3}$, by Lemma 2. If $\beta=(u+v \sqrt{-3}) / 2$, with $u \equiv v(\bmod 2)$, then $1=\operatorname{Tr}(\alpha)=\operatorname{Tr}\left(j \beta^{3}\right)$ yields $-8=\Delta(u, v)=u\left(u^{2}-9 v^{2}\right)+9 v\left(u^{2}-v^{2}\right)$ and $1=\operatorname{Tr}(\alpha)=\operatorname{Tr}\left(j^{2} \beta^{3}\right)$ yields $-8=\Delta(u,-v)$. If $U=(u-3 v) / 2$ and $V=(u+3 v) / 2$, then $\beta=\left(U+V+\frac{V-U}{3} \sqrt{-3}\right) / 2, \Delta(u, v)=-8\left(U^{3}-3 U V^{2}-V^{3}\right) / 3$ and $\Delta(u,-v)=$ $-8\left(V^{3}-3 V U^{2}-U^{3}\right) / 3$. Hence, $U^{3}-3 U V^{2}-V^{3}=3$ or $V^{3}-3 V U^{2}-U^{3}=3$, which implies $(U, V) \in\{(-1,-1),(2,-1),(-1,2)\}, \beta=-1,-j$ or $-j^{2}, \beta^{3}=-1$ and the desired result. Indeed, this is a Thue equation associated with the real cyclic cubic field $K=\mathbf{Q}(\alpha)$ of conductor 9, ring of algebraic integers $\mathbf{Z}[\alpha]$ and fundamental units $\eta_{1}=\alpha$ and $\eta_{2}=\alpha^{2}-\alpha-2$, where $\alpha^{3}-3 \alpha-1=0$ (we can also write this equation as $N(U-\alpha V)=3$, i.e. $U-\alpha V$ must be associated with $-1-\alpha$, of norm 3). Now, by using Bilu and Hanrot's method (see $[\mathrm{BH}]$ ), such a Thue equation can easily be solved by program packages like Kash (see [DFKPRW, Section 7.3]). 
It remains to deal with the prime $p=2$.

Lemma 4. Let $\mathbf{k}$ be a quadratic number field. If $\alpha \in \mathbf{A}_{\mathbf{k}}$, then $\alpha$ is a square in $\mathbf{A}_{\mathbf{k}}$ if and only if there exists $A \in \mathbf{Z}$ such that $N(\alpha)=A^{2}$ and such that $\operatorname{Tr}(\alpha)+2 A$ is a square in $\mathbf{Z}$. If $\mathbf{k}$ is imaginary, we may assume that $A \geq 0$.

Proof. If $\alpha=\beta^{2}$, then $N(\alpha)=A^{2}$ with $A=N(\beta)$ (hence $A \geq 0$ if $\mathbf{k}$ is imaginary), and $\operatorname{Tr}(\alpha)+2 A=\operatorname{Tr}(\beta)^{2}-2 N(\beta)+2 A=\operatorname{Tr}(\beta)^{2}$ is a square. Conversely, if $N(\alpha)=A^{2}$ and $\operatorname{Tr}(\alpha)+2 A=B^{2}$ are squares, then $\alpha^{2}-\operatorname{Tr}(\alpha) \alpha+N(\alpha)=0$ yields $\alpha^{2}-\left(B^{2}-2 A\right) \alpha+A^{2}=0$, and $\alpha=\left(\left(B \pm \sqrt{B^{2}-4 A}\right) / 2\right)^{2}$ is a square $\mathbf{A}_{\mathbf{k}}$.

\section{Proof of Theorem 1}

Set $\mathbf{k}=\mathbf{Q}\left(\sqrt{1-4 U^{n}}\right)$ and $\alpha=\left(1+\sqrt{1-4 U^{n}}\right) / 2 \in \mathbf{A}_{\mathbf{k}}$. Then $\operatorname{Tr}(\alpha)=1$ and $\alpha$ is not a unit in $\mathbf{A}_{\mathbf{k}}$ (for $N(\alpha)=U^{n}>1$ ). Since $\operatorname{Tr}(\alpha)=\alpha+\sigma(\alpha)=1$, the principal ideals $(\alpha)$ and $(\sigma(\alpha))$ are relatively prime and their product $(U)^{n}$ is an $n$th power. Therefore, $(\alpha)=\mathbf{I}^{n}$ is the $n$th power of some ideal $\mathbf{I}$ of $\mathbf{A}_{\mathbf{k}}$, and the ideal class of $\mathbf{I}$ is of order dividing $n$. Assume that it is not of order $n$. Then $\mathbf{I}^{n / p}=(\beta)$ is principal for some prime $p \geq 2$ dividing $n$. Hence $(\alpha)=\left(\beta^{p}\right)$ and $\alpha$ is associated with $\beta^{p}$, a $p$ th power in $\mathbf{A}_{\mathbf{k}}$. By Proposition 3, we have $p=2$, which proves the first point of Theorem 1. Finally, assume that $U \geq 3$ is odd and that $\alpha$ is associated with a square. Since $\mathbf{k} \neq \mathbf{Q}(\sqrt{-1}), \alpha$ or $-\alpha$ is a square. Now, if $\alpha$ is a square, then we know by Lemma 4 that $1+2 U^{n / 2}$ is a square. But since $U$ is odd, we know that $1+2 U^{n / 2} \equiv 3(\bmod 4)$, so $\alpha$ cannot be a square. Thus, $-\alpha$ is a square, and we conclude that $-1+2 U^{n / 2}$ is a square by Lemma 4. It follows that for any prime $p \geq 3$ dividing $U,-1 \equiv-1+2 U^{n / 2}(\bmod p)$ is a square $\bmod p$; that is, $p \equiv 1(\bmod 4)$, which proves the second point of Theorem 1 .

Remark 5 . We do not need the results on the Thue equation $U^{3}-3 U V^{2}-V^{3}=3$ to prove that if 3 divides an odd integer $U \geq 3$, then $n>1$ divides the class number of the imaginary quadratic number field $\mathbf{k}=\mathbf{Q}\left(\sqrt{1-4 U^{n}}\right)$, for in that situation we have $\mathbf{k} \neq \mathbf{Q}(\sqrt{-3})$.

\section{REFERENCES}

[AC] N. C. Ankeny and S. Chowla, On the divisibility of the class number of quadratic fields, Pacific J. Math. 5 (1955), 321-324. MR0085301 (19:18f)

[BH] Y. Bilu and G. Hanrot, Solving Thue equations of high degree, J. Number Theory 60 (1996), 373-392. MR.1412969 (97k:11040)

[Co] J. H. E. Cohn, On the class number of certain imaginary quadratic fields, Proc. Amer. Math. Soc. 130 (2002), 1275-1277. MR.1879947 (2002j:11127)

[DFKPRW] M. Daberkow, C. Fieker, J. Klüners, M. Pohst, K. Roegner, M. Schörnig and K. Wildanger, KANT V4, J. Symbolic Comput. 24 (1997), 267-283. MR.1484479 (99g:11150)

[GR] B. H. Gross and D. E. Rohrlich, Some results on the Mordell-Weil group of the Jacobian of the Fermat curve, Inventiones Math. 44 (1978), 201-224. MR0491708 (58:10911)

[Ki] Y. Kishi, Note on the divisibility of the class number of certain imaginary quadratic fields, Glasgow Math. J. 51 (2009), 187-191. MR2471686 
[Mur] M. Ram Murty, Exponents of class groups of quadratic fields, Topics in number theory (University Park, PA, 1997), 229-239, Math. Appl., 467, Kluwer Acad. Publ., Dordrecht, 1999. MR1691322 (2000b:11123)

[So] K. Soundararajan, Divisibility of class numbers of imaginary quadratic fields, J. London Math. Soc. (2) 61 (2000), 681-690. MR1766097 (2001i:11128)

Institut de Mathématiques de Luminy, UMR 6206, 163, avenue de Luminy, Case 907, 13288 Marseille Cedex 9, France

E-mail address: loubouti@iml.univ-mrs.fr 